> Les vésicules extracellulaires, sécrétées spontanément ou en réponse à un stress par tous les types cellulaires, sont proposés comme des biothérapies alternatives aux thérapies cellulaires et aux nanomédicaments synthétiques. Leurs atouts logistiques (stockage, stabilité, disponibilité, tolérance), leur capacité à franchir les barrières biologiques, à délivrer leurs contenus (protéines, lipides et acides nucléiques) pour modifier leurs cellules cibles, ainsi que leurs activités immunomodulatrice et régénérative, suscitent un intérêt grandissant pour un très large spectre de maladies. Cette synthèse présente les défis qui restent à relever pour appliquer ces biothérapies en clinique. Quelques applications prometteuses dans les domaines du cancer et de la médecine régénérative seront proposées. <

Les vésicules extracellulaires $(V E)$ sont des vésicules membranaires de quelques dizaines à quelques centaines de nanomètres, sécrétées par les cellules de manière physiologique ou en réponse à un stress. Les $V \varepsilon$ circulent dans le tissu environnant puis dans l'ensemble des fluides biologiques, atteignant ainsi des tissus distants. Elles jouent un rôle de vecteurs pour la communication intercellulaire. Leurs compositions lipidique et protéique diffèrent selon la cellule productrice, son environnement et les stimulus qui conduisent à leur sécrétion. Les $V \varepsilon$ véhiculent des biomolécules, telles que des facteurs trophiques ou de différenciation, des oncogènes ou des acides nucléiques. L'internalisation des VE peut modifier le phénotype des cellules receveuses, leur métabolisme et leurs fonctions, moduler leur croissance et leur sécrétion, ou orienter la différenciation cellulaire et la réponse immunitaire [1]. Les $V \varepsilon$ régulent ainsi de nombreux processus physiopathologiques, comme l'inflammation, l'angiogenèse, le

Vignette (๔ Guillaume van Niel, Aurélie di Cicco, Graça Raposo, Daniel Levy).

\section{Applications thérapeutiques des vésicules extracellulaires}

Kelly Aubertin ${ }^{1}$, Max Piffoux ${ }^{2,3}$, Anna Sebbagh ${ }^{1}$, Jeanne Gauthier ${ }^{4}$, Amanda K.A. Silva ${ }^{1}$, Florence Gazeau ${ }^{1}$

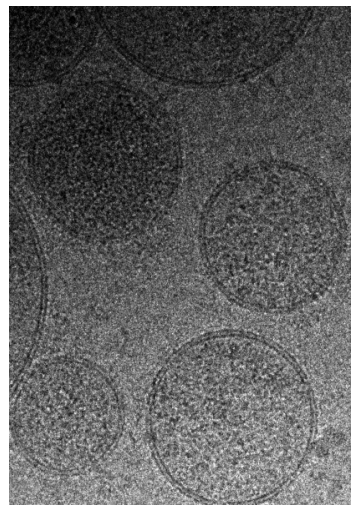

${ }^{1}$ Laboratoire matière et systèmes complexes (MSC), université de Paris, CNRS UMR7057, 45 rue des Saints Pères, 75006 Paris, France ${ }^{2}$ Service d'Oncologie médicale, Centre Léon Bérard, Lyon, France. ${ }^{3}$ Oncologie médicale, Institut de Cancérologie des Hospices Civils de Lyon (IC-HCL), CITOHL, Centre Hospitalier Lyon-Sud, Lyon, France.

${ }^{4} \mathrm{AP}-\mathrm{HP}$, Université de Paris, Paris, Fance.

métabolisme énergétique, l'immunité, florence.gazeau@u-paris.fr

la coagulation, le cancer, le vieillissement, la régénération tissulaire, ou encore la réponse à des pathogènes, à des polluants ou des substances toxiques.

Par leurs activités biologiques et leur présence dans les différents biofluides, les VE représentent non seulement de petites sondes diagnostiques circulantes accessibles par «biopsie liquide », mais aussi des nano-outils thérapeutiques de choix. En effet, leurs capacités intrinsèques à franchir les barrières biologiques (par exemple la barrière hémato-encéphalique [2]) et à délivrer leurs contenus à des cellules spécifiques pour en moduler l'activité, en font d'excellents candidats comme vecteurs pour les thérapies ciblées et la médecine régénérative $[3,4]$. Les perspectives en médecine personnalisée sont nombreuses grâce à leur faible immunogénicité et aux possibilités de leur ingénierie (chargement de petites molécules, enrichissement par des protéines ou des ARN thérapeutiques, ajouts d'agent de ciblage) [5]. Elles permettent d'égaler la versatilité des vecteurs synthétiques tout en profitant d'avantages discriminants liés à leur origine cellulaire. Modifiées ou non, les VE sont proposées comme une alternative plus sûre, et logistiquement plus simple, à l'administration de cellules vivantes, telles que les cellules souches, dont elles possèdent les propriétés régénératives, immunomodulatrices et anti-inflammatoires. On assiste ainsi à l'émergence de biothérapies «sub-cellulaires» à base de VE dans de très nombreuses indications, particulièrement en médecine régénérative. Les verrous associés à leurs applications cliniques restent cependant nombreux, concernant notamment la caractérisation des $V E$, la production à grande échelle, l'ingénierie, le contrôle qualité et les aspects réglementaires [6]. En France, où la 
recherche sur les $V \varepsilon$ a été et reste pionnière, une filière industrielle des biothérapies à base de VE émerge, soutenu par l'État et adossée à un fort réseau de compétences académiques.

\section{Biogenèse et bioproduction de vésicules extracellulaires (VE) à visée thérapeutique}

\section{Biogenèse}

Plusieurs mécanismes de formation des VE ont été décrits, à l'origine de diverses sous-populations de VE, notamment, les exosomes (50-150 $\mathrm{nm}$ ) libérés par fusion des corps multivésiculaires avec la membrane plasmique, les microvésicules ou ectosomes (100-1000 nm) formées par bourgeonnement de la membrane plasmique, ou encore les corps apoptotiques (1-5 $\mu \mathrm{m})$. Ces sous-populations de VE partagent un bon nombre de biomolécules (lipides, protéines, acides nucléiques) et leur distinction en terme de taille ou de composition n'est pas aisée [3]. Le consensus actuel, particulièrement pour les applications thérapeutiques, considère un continuum de $V \varepsilon$, avec des sous-classes établies selon la taille [7], ou une fraction du sécrétome cellulaire enrichie en $\vee \varepsilon$ (vesicular secretome fraction). Parmi les facteurs qui influencent la composition des VE, figurent le type de cellules dont elles sont issues ainsi que les conditions de culture cellulaire utilisées pour leur production (conditionnement ou priming cellulaire).

\section{Bioproduction de Vع}

Selon l'effet thérapeutique recherché, les VE peuvent être produites à partir de culture de différents types cellulaires (macrophages, plaquettes sanguines, cellules dendritiques, cellules stromales mésenchymateuses [CSM], cellules souches pluripotentes induites [IPS], etc.) ou purifiées à partir de biofluides [3, 4]. Les CSM humaines isolées et amplifiées à partir de la moelle osseuse, du tissu adipeux ou de sang de cordon ombilical, sont des sources cellulaires de choix car leurs effets régénératifs et immunomodulateurs ont été largement démontrés et attribués aux facteurs paracrines que leurs vésicules transportent $[3,8]$. Les cellules peuvent provenir du patient traité (autologue) ou d'un ou plusieurs donneurs (allogénique) pour constituer une banque de cellules permettant de générer des lots de vésicules homogènes. Grâce à la source allogénique de $V \varepsilon$, se dessine la possibilité de pouvoir traiter plusieurs patients avec le même lot de vésicules, et donc d'obtenir une biothérapie accessible et disponible à la demande. Actuellement, des stratégies d'immortalisation des cellules sécrétrices sont proposées afin d'augmenter leur stabilité phénotypique au cours des divisions et ainsi accroître les rendements et la reproductibilité des lots de vésicules produits à partir d'une même source. Les cellules sécrétrices peuvent par ailleurs être modifiées génétiquement pour enrichir les vésicules qu'elles produisent, en protéines ou en ARN d'intérêt thérapeutique, ou pour augmenter leur capacité de ciblage en modifiant leur composition membranaire [5]. Malgré la grande diversité des sources et des modifications cellulaires, un défi majeur de la bioproduction de VE est d'atteindre une échelle compatible avec une utilisation clinique, tout en assurant la qualité et la reproductibilité des lots obtenus et en minimisant les coûts. Si les cellules produisent spontanément les $V \varepsilon$, des bioprocédés sont proposés pour augmenter les rendements de production : accroissement du nombre de cellules cultivées en parallèle ; augmentation du temps de culture et/ou du nombre des VE émises par cellule. Certaines techniques de production massive sont disponibles commercialement (par exemple la culture sur fibres creuses), mais les VE produites et leurs effets thérapeutiques nécessitent d'être précisément caractérisés [9]. Le nombre de VE générées par cellule peut être augmenté par différents types de stimulus, notamment mécanique [10-13], hypoxique, chimique $[14,15]$, et/ou par une carence en nutriments. Ces stimulus miment souvent ceux induits lors de processus physiopathologiques au cours desquels la vésiculation est initiée. Par exemple, des contraintes anormales de cisaillement, comme celles rencontrées par les cellules au sein des vaisseaux, peuvent induire une augmentation de la tension membranaire et activer l'exocytose [11, 16]. Notre équipe a breveté une méthode de production par turbulence dans laquelle le stress mécanique induit une vésiculation environ dix fois plus importante et dix fois plus rapide (quelques heures) que dans une culture en flasque et milieu carencé. Ce procédé à fort rendement, adaptable à différentes tailles de bioréacteurs en condition de bonnes pratiques de laboratoire (BPL ou GMP, en anglais), est en cours d'industrialisation par l'entreprise EVerZom'.

Si l'augmentation de production, la facilité, le temps, les rendements, la reproductibilité et les coûts de production sont des éléments clés pour le transfert industriel, les procédés de vésiculation par conditionnement cellulaire offrent aussi l'opportunité de moduler le contenu des VE et d'adapter leurs activités biologiques à des indications données. À l'instar de ce qui se passe dans nos tissus, la sécrétion de $V \varepsilon$ est une réponse de la cellule à un stress, en exportant et transférant des signaux biologiques. La composition des VE peut ainsi dépendre de l'amplitude de contraintes mécaniques [17], d'un stress chimique [18] ou de facteurs appliqués aux cellules lors de la production. En fonction de l'indication recherchée, il est possible d'imaginer et d'optimiser un procédé de production pour améliorer l'efficacité des VE [10, 19]. Un des défis actuels est donc de comprendre les relations complexes qui existent entre conditionnement cellulaire durant la production, identité des VE (leur signature biologique et physique) et activités thérapeutiques. Des méthodes de criblage haut débit (high-throughput screening) et multimodales sont donc nécessaires pour caractériser les $V \varepsilon$ et pour comparer leurs propriétés thérapeutiques

\footnotetext{
http://everzom.com
} 
par des tests bien définis (potency assays), encore peu prédictifs d'une activité in vivo. Se pose également la question des étapes et des méthodes d'isolement et/ou de purification pour séparer les différentes populations de VE des facteurs solubles et des agrégats protéiques présents dans le sécrétome cellulaire [20]. La diversité des méthodes utilisées rend difficile les comparaisons entre les études. On observe ainsi une très grande hétérogénéité des dosages de VE dans les essais précliniques et cliniques (quantification en nombre d'objets, en quantité de protéine totale, en protéine spécifique, en cargo d'intérêt, etc.) [21]. Bien que l'utilisation des $V \varepsilon$ dans le domaine thérapeutique soit un domaine en plein essor, leur transfert vers la clinique n'en est qu'à ses débuts, sans règlementation spécifique. Depuis quelques années, plusieurs groupes de travail tâchent d'uniformiser les techniques de caractérisation et de délimiter des critères de contrôle qualité, de sûreté et d'efficacité des VE et des produits thérapeutiques associés. Ces efforts permettent de discuter avec les agences de santé pour définir un cadre réglementaire plus précis et adapté. Citons ainsi la Regulatory affairs task force de I'International Society for Extracellular Vesicles (ISEV) 2, I'Exosomes committee de I'International Society for Gene and Cell Therapy (ISCT) ${ }^{3}$, ou le groupe de travail français Extracellular vesicle translation to clinical perspective (EVOLVEFrance) $[53]^{4}$.

\section{Mécanismes d'action des $V \varepsilon$ : propriétés intrinsèques}

L'internalisation des VE par les cellules réceptrices repose sur des mécanismes de fusion entre la membrane vésiculaire et la membrane plasmique [22] et/ou sur plusieurs voies d'endocytose [3]. Les VE sont naturellement équipées pour être reconnues par des récepteurs membranaires puis internalisées par les cellules qu'elles ciblent. Les mécanismes de transfert du contenu intravésiculaire dans le cytoplasme de la cellule restent mal connus, mais ils reposeraient sur la fusion des VE internalisées avec la membrane des endosomes. Ce mécanisme, utilisé par de nombreux virus, est favorisé par le $\mathrm{pH}$ acide des endosomes [22].

Les VE issues de CSM ont des propriétés immunomodulatrices et ciblent préférentiellement des zones inflammatoires ou tumorales [12, 23]. Certaines VE jouent ainsi un rôle dans la restauration de la fonction tissulaire et l'homéostasie cellulaire à travers des processus tels que la prolifération et la différenciation cellulaire, ou la sénescence [4, 24]. Quelques mécanismes d'action, proposés pour les $V \varepsilon$, et comparés à ceux des cellules stromales dont elles sont issues, sont résumés dans la Figure 1 dans le contexte de maladies inflammatoires gastro-intestinales [24]. II reste cependant difficile de déterminer quels mécanismes sont pertinents selon le contexte pathologique.

2 https://www.isev.org/index.php?option=com_content\&view=article\&id=60: regulatory-affairs-taskforce\&catid $=20$ : site-content

${ }^{3}$ https://www.isctglobal.org/about/isct-committees/exosomes

${ }^{4}$ https://evolve-france.u-paris-sciences.fr/ [53].

\section{Les $V \varepsilon$ pour la médecine régénérative}

Ces dernières décennies ont vu l'émergence de biothérapies fondées sur l'administration de cellules vivantes, souvent des cellules souches, afin de favoriser la réparation d'un organe ou d'un tissu défaillant, le protéger d'une dégénérescence accélérée, ou pour moduler un processus inflammatoire [25] $(\rightarrow)$.

On peut citer, par exemple, la pre- $(\rightarrow)$ Voir Synthèse de M. Maumus et al., $\mathrm{m} / \mathrm{s}$ $n^{\circ} 12$, décembre 2018 , page 1092

\section{mière autorisation européenne de}

mise sur le marché (AMM), en 2018, de CSM allogéniques issues de tissu adipeux (ADSC, pour adipose-derived stem cells) (alofisel/darvadstrocel) ${ }^{5}$ pour le traitement des fistules péri-anales complexes chez l'adulte atteint de la maladie de Crohn, une maladie inflammatoire chronique de l'intestin ( $\mathrm{MICl}$ ). Le mécanisme d'action proposé est une diminution de la prolifération des lymphocytes activés et de la libération de cytokines proinflammatoires, permettant une meilleure cicatrisation du trajet fistulaire.

Cependant, dans différents contextes, les cellules administrées ne survivent pas. Les effets thérapeutiques que l'on observe ont donc été attribués à des facteurs paracrines, en particulier aux VE que ces cellules sécrètent pendant les quelques jours où elles sont proches de la lésion à traiter. Ce constat a suscité de nombreuses études précliniques et quelques essais cliniques (Tableau I) dans lesquels les VE ont été injectées à la place des cellules dont elles sont issues. Dans la grande majorité des cas, ces essais se sont traduits par un effet thérapeutique régénératif, immunomodulateur et/ou protecteur de même ampleur que celui produit par une injection des cellules souches parentales. Les maladies qui ont été ciblées sont aussi diverses que la maladie du greffon contre l'hôte (GvH) [26] après une transplantation de moelle osseuse chez les patients atteints de leucémie, la préservation d'organes avant leur transplantation, des maladies auto-immunes ou inflammatoires de l'intestin, l'accident vasculaire cérébral ou les traumatismes crâniens [27], l'arthrose et la régénération des disques intervertébraux [28], le syndrome de détresse respiratoire, les brûlures ou les myopathies cardiaques [29]. Récemment, plusieurs essais cliniques ont été lancés chez des patients ayant contracté la Covid-19 (coronavirus disease 2019), pour induire la régénération des tissus pulmonaires détruits par le virus et la résolution de l'inflammation produite [4].

\footnotetext{
5 https://www.has-sante.fr/jcms/c_2904636/fr/alofisel-darvadstrocel-therapiecellulaire
} 


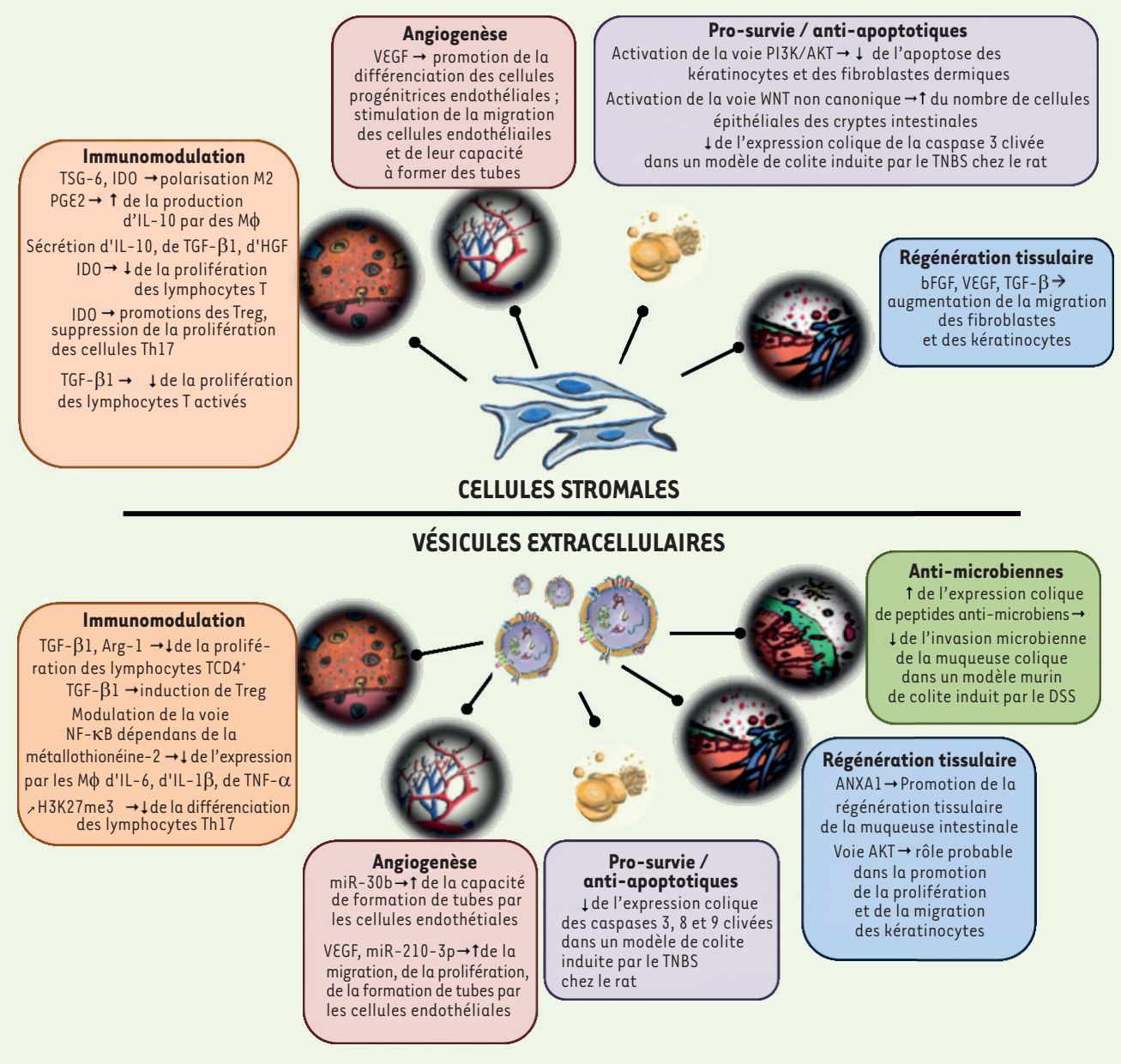

Figure 1. Quelques mécanismes d'action possibles des cellules stromales et de leurs $V \varepsilon$ dans une contexte d'inflammation et de régénération tissulaire [24]. TSG-6 : tumor necrosis factor-stimulated gene 6 ; IDO : indoleamine 2,3-dioxygénase ; PGદ2 : prostaglandine $\varepsilon 2$; MФ : macrophage ; IL-10 : interleukine 10 ; TGF- $\beta$ : transforming growth factor $\beta$; HGF : hepatocyte growth factor ; Treg : lymphocyte T régulateur ; Th17 : lymphocyte T auxilliaire (helper) 17 ; VEGF : vascular endothelial growth factor ; PI3K : phosphoinositide 3-kinase ; bFGF : basic fibroblast growth factor ; Arg-1 : arginase 1 ; NF-KB : nuclear factor-kappa B; IL-6 : interleukine 6 ; IL-1 $\beta$ : interleukine $1 \beta$; TNF- $\alpha$ : tumor necrosis factor $\alpha$; H3K27me3 : triméthylation de la lysine 27 de l'histone 3 ; miR-30b : micro-ARN 30b ; miR-210-3p : micro-ARN 210-3p; TNBS: acide 2,4,6-trinitrobenzène sulfonique ; ANXAl : annexine Al; DSS : sel de sodium au sulfate de dextrane.

Les avantages des biothérapies vésiculaires, par rapport aux thérapies cellulaires, sont non seulement logistiques (conservation et disponibilité immédiate de lots de VE allogéniques), mais également biologiques, avec une capacité de pénétration tissulaire des $V \varepsilon$ qui peut être favorable par rapport à celle des cellules, une absence de réplication ou de différenciation qui pourraient autrement être dommageables, une diminution du risque d'occlusion vasculaire, une induction moindre de réponse immunitaire pouvant être de plus modulée par ces VE.

Plusieurs verrous freinent encore le transfert clinique des thérapies vésiculaires, tels que le choix d'un mode d'administration et la détermination de la dose utile [21]. Comme pour les thérapies cellulaires, l'administration locale dans le tissu lésé est souvent privilégiée du fait de la demi-vie extrêmement courte des Vદ dans la circulation.
Néanmoins, le devenir des VE administrées et leurs cibles cellulaires restent mal connus, même si des effets immunomodulateur, anti-inflammatoire, antifibrotique et pro-angiogénique ont été observés dans différents contextes, y compris après une injection par voie intraveineuse. Pour prolonger la rétention des VE au voisinage du tissu lésé, notre équipe et celle de Gabriel Rahmi, à l'hôpital européen Georges Pompidou (PARCC, HEGP) a proposé d'associer les VE à un gel thermosensible, le poloxamère 407 (ou PF-127 pour Pluronic F-127) (Figure 2) [30]. Ce polymère a la propriété intéressante d'être liquide à température ambiante, ce qui facilite la dispersion des $\vee \varepsilon$ et le remplissage du trajet fistuleux souvent sinueux. II se solidifie à température 
corporelle, permettant de conserver les VE sur le site de leur injection [13] et joue un rôle occlusif tout en délivrant de manière homogène et durable les VE lors de la dilution du gel par les sécrétions. L'association des VE issues d'ADSC et de ce polymère s'est révélée particulièrement efficace pour la cicatrisation dans différents modèles de fistules digestives chez le petit et le gros animal : fistule colo-cutanée chez le rat [13] et fistule œsophago-cutanée chez le porc [31]. Le PF-127 per se a aussi un effet pro-cicatrisant dans ces deux modèles animaux et chez l'homme [32], avec une réduction du diamètre fistuleux et du volume des sécrétions ; cependant, ces effets sont améliorés par l'activité biologique des $V \varepsilon$, permettant d'obtenir une fermeture complète des fistules dans le modèle de fistule de l'œsophage chez le porc [31]. Des études sont en cours pour étendre ces résultats à d'autres types de fistules, consécutives par exemple, à une maladie de Crohn, et pour déterminer si la thérapie vésiculaire peut être une alternative avantageuse en termes de coût, de tolérance et d'efficacité thérapeutique à la thérapie cellulaire (alofisel/darvadstrocel) déjà sur le marché mais non remboursée en France pour des problèmes de coût et de logistique. L'association du gel aux VE issues d'ADSC a également montré son efficacité pour la prévention des sténoses de l'œsophage, après dissection de la sous-muqueuse par endoscopie chez le porc [33]. À noter que les VE utilisées dans ces dernières études avaient été produites par le procédé de production haut débit utilisant la turbulence (stress mécanique), compatibles avec des conditions de grade BPF/GMP. La startup Evora Biosciences a récemment été créée pour tenter de transférer au patient ces thérapies vésiculaires dans ces indications, notamment les fistules digestives.

Une des applications des VE les plus avancées en France, proposée par les équipes de Philippe Menasché (HEGP) et de Jérôme Larghero (Hôpital Saint-Louis), concerne le traitement de l'insuffisance cardiaque chronique. Après différents essais d'injection dans le myocarde de cellules différenciées en cardiomyocytes à partir de cellules souches embryonnaires, il est apparu que l'effet cardio-protecteur de ces cellules reposait sur leur sécrétome. Dans des modèles murin [34] ou por$\operatorname{cin}[35]$, I'utilisation de VE, à la place des cellules d'origine, a reproduit les effets bénéfiques observés avec les cellules parentales, avec l'induction de l'angiogenèse via la présence, au sein des $V E$, de microARN ciblant des voies de signalisation impliquées dans la formation vasculaire et dans la prolifération cellulaire. Les VE issues de progéniteurs cardiaques sont également susceptibles d'induire l'activation de voies de régénération intrinsèques du myocarde [36]. Une modulation de l'état inflammatoire et du microenvironnement immunologique au sein du myocarde lésé est également observée, avec la diminution des taux de cytokines pro-infllammatoires (IL[interleukine]-l $\alpha$ et IL-6) et l'augmentation de ceux d'IL-10, cytokine anti-inflammatoire, ainsi qu'avec une polarisation des macrophages privilégiant le type M2 (anti-inflammatoire) plutôt que Ml (inflammatoire). En vue d'un usage clinique, notons que les $V \varepsilon$ dérivant de progéniteurs cardiaques n'expriment pas ou peu de récepteurs immunitaires régulateurs, tels que les molécules du complexe majeur d'histocompatibilité (CMH) de classe I, le ligand de PD-1 (programmed cell death 1 ligand, PD-LI) ou ICOS-L (inducible costimulator-ligand) et donc, contrairement à une thérapie cellulaire classique, ne provoquent pas de réaction immunitaire cellulaire ou humorale [37].

Dans un autre contexte, celui des maladies ostéoarticulaires, les travaux de l'équipe de Christian Jorgensen et Danièle Noël (Institut de médecine régénératrice et de biothérapie, CHU de Montpellier) ont mis en évidence l'effet thérapeutique des VE issues de CSM, en application locale, en particulier dans des modèles précliniques d'arthrose, avec une augmentation des marqueurs anaboliques (collagène de type II et aggrécane, composants essentiels du cartilage) associée à une inhibition des marqueurs d'inflammation (synthase inductible de l'oxyde nitreux - iNOS) et d'apoptose [38]. Cette équipe a également démontré l'intérêt thérapeutique de ces $\vee \varepsilon$ pour la thérapie de l'arthrite inflammatoire et de la sclérodermie systémique $[39,40]$.

Toujours en application locale, l'équipe d'Eva Rohde (Paracelsus Medical University) à Salzbourg, en Autriche, a très récemment réalisé chez un patient la première implantation cochléaire de $V \varepsilon$ issues de cellules souches de cordon ombilical (de grade clinique car produites et caractérisées dans des conditions GMP), dans l'objectif de réduire l'inflammation liée à un implant cochléaire [41]. Cette première chez l'homme faisait suite à une étude préclinique montrant les propriétés immunomodulatrices de ces $V \varepsilon$ sur des lymphocytes $T$ et des cellules microgliales, leur activité neuroprotectrice, et leur capacité à atténuer la perte d'audition et la destruction de cellules ciliées auditives après un traumatisme sonore chez la souris [42]. Cette intervention révèle ainsi la faisabilité d'une administration adjuvante de $V \varepsilon$ dans la cochlée lors de la mise en place de l'implant, sans effets secondaires sur 24 mois, et avec une amélioration des capacités auditives de l'oreille traitée comparable à celle obtenue avec l'implant seul. Grâce à la production et la qualification de lots cliniques des VE utilisées, les essais de phases I, puis II/III pourront être programmés, après la réalisation préalable, dans un modèle préclinique, des tests de toxicité (immunotoxicité) et de pharmacocinétique. Le Tableau / recense les différents essais cliniques en cours utilisant les $\vee \varepsilon$ pour la médecine régénérative $[3,4]^{6}$.

\section{Les VE comme vecteurs d'agent thérapeutique}

L'encapsulation d'un principe actif dans un nanovecteur, qu'il soit synthétique ou biogénique, a pour but de protéger l'agent thérapeutique d'une dégradation ou d'une réponse immunitaire, de concentrer et transporter des agents hydrophiles ou hydrophobes, d'en

\footnotetext{
${ }^{6}$ https://clinicaltrials.gov/ct2/home
} 


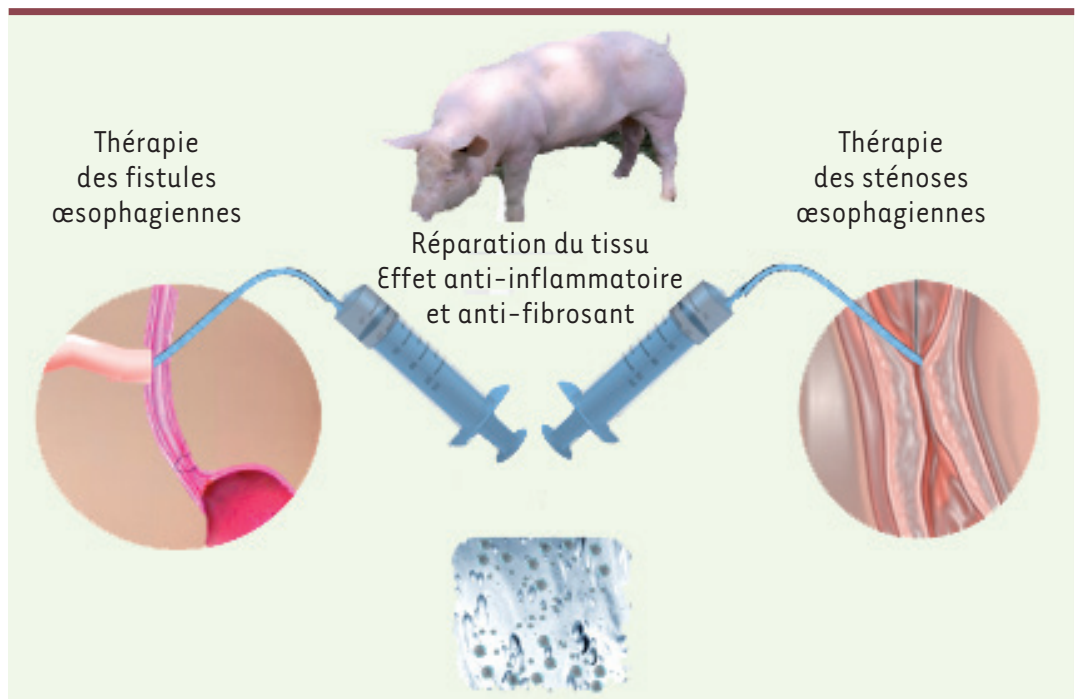

Poloxamer 407 + vésicules extracellulaires (issues des cellules stromales du tissu adipeux)

modifier la biodistribution, de protéger l'organisme de leur toxicité et de favoriser une délivrance ciblée. Les liposomes figurent parmi les vecteurs synthétiques les plus utilisés, certains ayant reçu une AMM. Toutefois, l'injection répétée de tels vecteurs peut entrainer une réaction immunitaire spécifique, et, malgré les nombreuses stratégies de ciblages actif ou passif, leur pharmacocinétique reste mal contrôlée. Les VE ou les vecteurs hybrides (par exemple, des VE fusionnées avec des liposomes) présentent des avantages en termes de biocompatibilité, de ciblage tissulaire, cellulaire et intracellulaire, et de délivrance de leurs cargos, en plus de leur activité biologique intrinsèque. Pour ajouter des agents exogènes aux VE (nanoparticules, petites molécules, ARN, agent de ciblage, protéines) ou les enrichir en protéines, lipides, micro-ARN, ARN messagers qui y sont naturellement présents, plusieurs stratégies d'ingénierie ont été développées $[5,10,43]$ :

Chargement «pré-production» par ingénierie de la cellule d'origine: l'agent thérapeutique est exprimé, surexprimé ou incorporé (petites molécules, nanoparticules) dans les cellules productrices avant d'initier la production de VE ;

Chargement «post-production»: I'agent thérapeutique est introduit dans les $V \varepsilon$ après leur production par incubation avec le principe actif, par modification de surface (telles que fonctionnalisation de la membrane de la VE par chimie-click), par fusion avec des liposomes ou des micelles préchargées avec le principe [44], par perméabilisation de la membrane (par électroporation, sonoporation, extrusion, etc) ;

Chargement «per-production»: il s'agit alors d'une encapsulation du principe actif au cours de la formation des $V \varepsilon$ ou dans des vésicules issues du fractionnement de membranes cellulaires (nano-ghosts [45]).

L'efficacité d'encapsulation et la capacité de chargement dépendent du principe actif et de ses propriétés physico-chimiques (taille, hydrophilicité, etc.), et de la méthode utilisée [43]. Celle-ci peut altérer l'intégrité des $V \varepsilon$ (perte de l'asymétrie membranaire, perte de contenu) et donc leur activité biologique.
Figure 2. Administration locale du gel thermosensible poloxamer 407 chargé des $V \varepsilon$ issues de cellules stromales mésenchymateuses (CSM) $d u$ tissu adipeux pour la cicatrisation de fistules digestives et pour la prévention des sténoses de l'œesophage (d'après [13, 30-33]).

Les VE, vecteurs d'agent thérapeutique en cancérologie...

L'un des domaines les plus actifs dans le développement de VE chargées est celui de la cancérologie (Tableau II). Une grande variété de sources cellulaires est utilisée, d'origine humaine (cellules dendritiques, CSM, cellules tumorales, cellules autologues, etc.), s'appuyant sur un tropisme tumoral ou/et une activité immunomodulatrice ou de vaccination, mais pouvant aussi être d'origine végétale ou bactérienne, ce qui permet de diminuer les coûts de bioproduction. Divers agents chimiothérapeutiques, comme la doxorubicine, la gemcitabine, le carboplatine, ou des petits ARN interférants (small interfering $R N A$ ) ont été encapsulés dans les VE.

Un photosensibilisateur activable par laser, la métatétra(hydroxyphényl)chlorine (mTHPC ou Foscan ${ }^{\circledR}$ ou Temoporfin), non cytotoxique en l'absence d'excitation lumineuse, et approuvé cliniquement pour une utilisation en photothérapie dynamique (PDT), a été encapsulé dans des VE de macrophages, de cellules endothéliales ou de CSM par simple incubation avec ces cellules avant la vésiculation. Des nanoparticules magnétiques, chargées avec le photosensibilisateur, rendait ces VE détectables en IRM et manipulables par des gradients de champ magnétique [46]. Comparé à la forme libre du mTHPC et à des liposomes contenant ce même agent (Foslip ${ }^{\circledR}$ ), les VE délivrent le mTHPC plus profondément dans des sphéroïdes tumoraux ; elles en modifient également la distribution tissulaire [47], qu'elles soient injectées par voie intraveineuse, en intra-tumoral ou en intrapéritonéal. Des VE issues de CSM produites par turbulence à haut débit et chargées de MTHPC ont été administrées dans le péritoine de souris présentant des métastases péritonéales d'origine colique ou ovarienne [12]. Ces $V \varepsilon$ permettent une augmentation très importante de la sélectivité intra-tumorale du mTHPC par rapport à sa formulation libre ou liposomale, ce qui permet de réduire la toxicité de la photothérapie dynamique sur les tissus sains, facteur limitant de son utilisation pour le traitement de métastases péritonéales. Les $V \varepsilon$ activées par laser induisent une réponse immuni- 


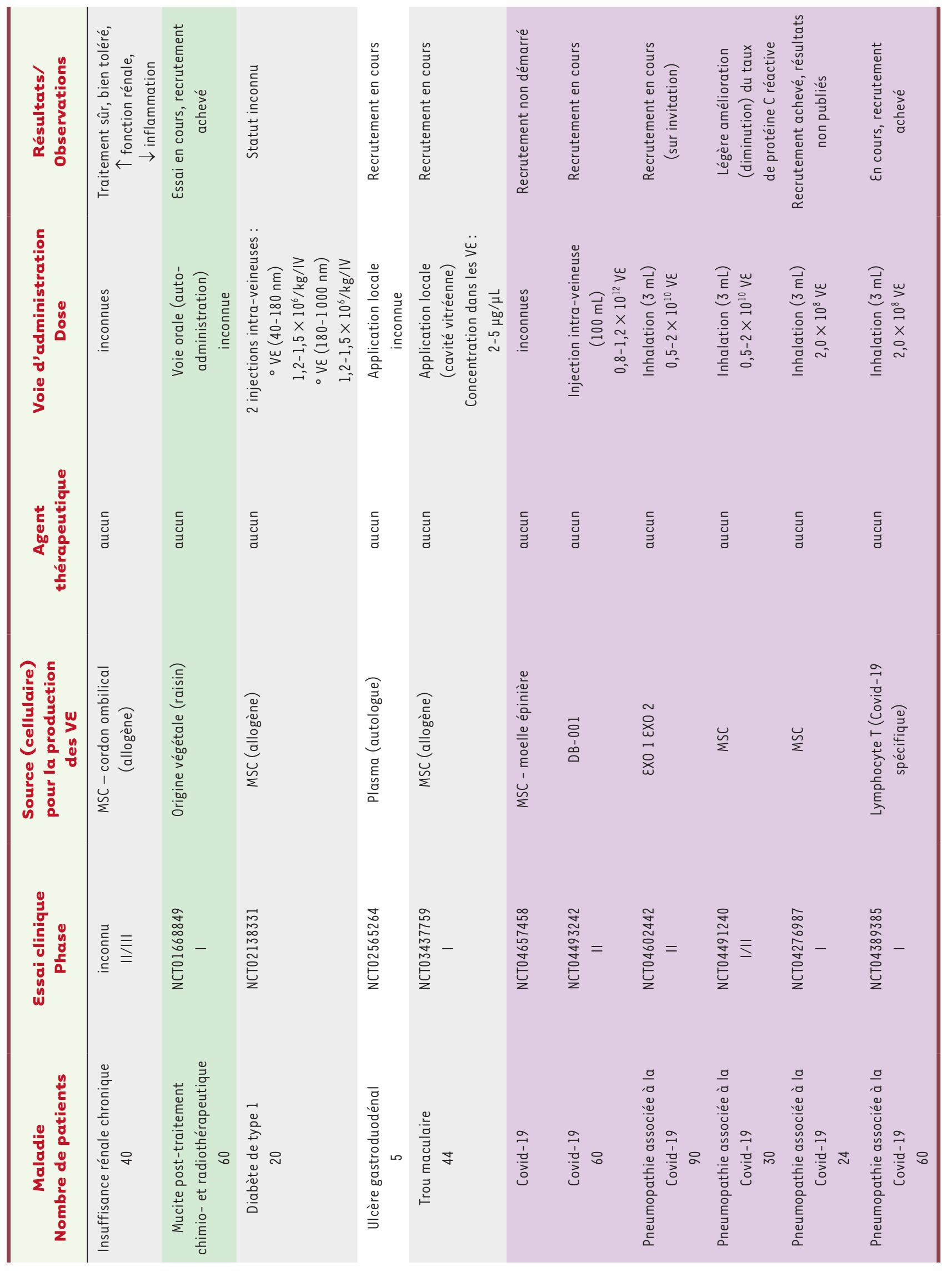




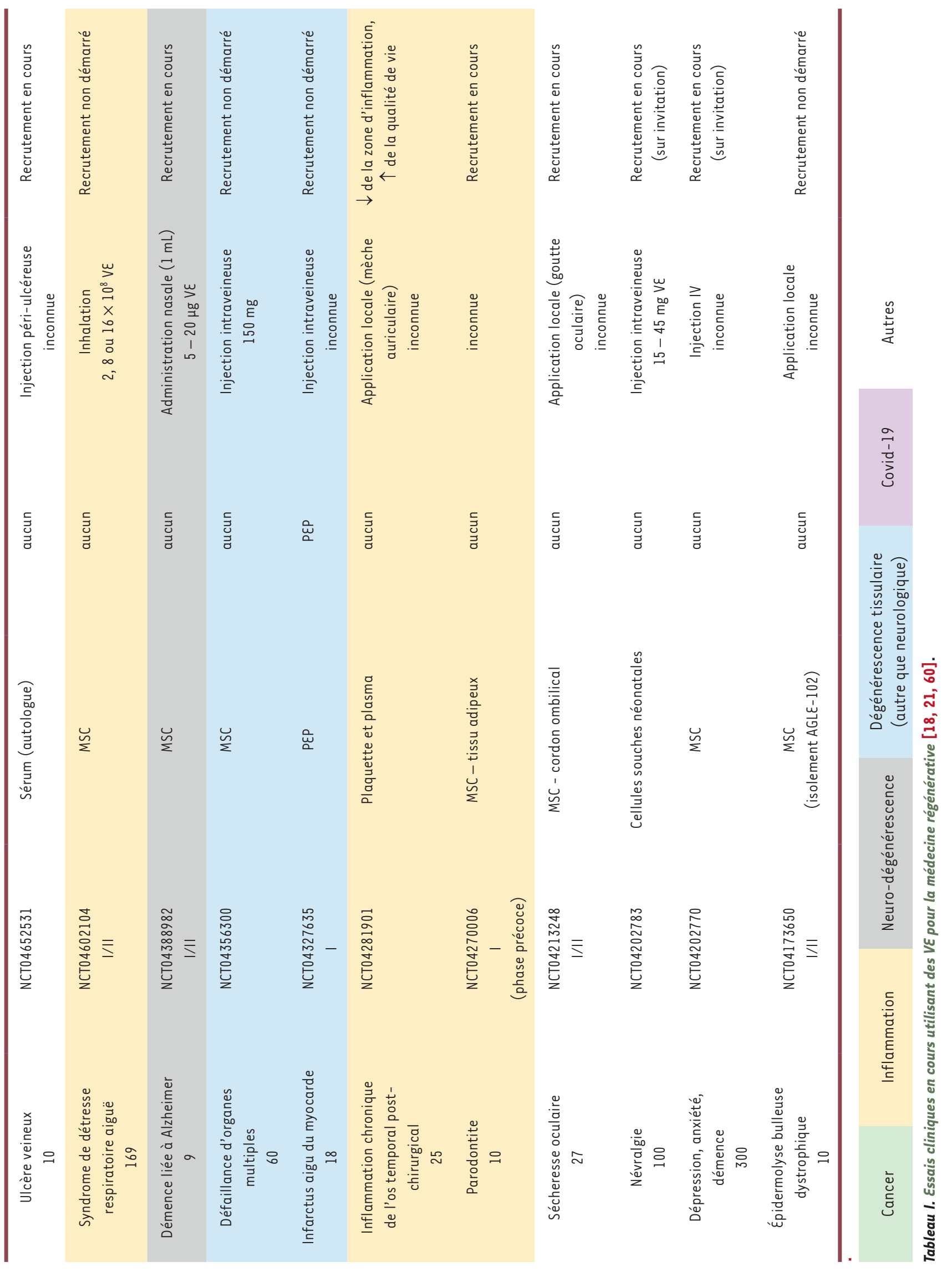


taire anti-tumorale (principalement des macrophages Ml pro-inflammatoires et des lymphocytes $\mathrm{T}^{\mathrm{C}} \mathrm{C} 8^{+}$cytotoxiques), une régression de la prolifération tumorale et un prolongement de la survie des souris supérieur aux liposomes. II s'agit donc d'un exemple de combinaison d'un ciblage tumoral et d'effets immunomodulateurs propres aux VE de CSM avec les effets cytotoxiques d'un photosensibilisateur, de manière contrôlée dans l'espace et dans le temps. Ces VE, compatibles avec une production de masse de grade clinique, pourraient constituer une nouvelle génération de photosensibilisateurs pour augmenter la précision de la photothérapie dynamique.

Très récemment, l'entreprise Codiak Biosciences, pionnière dans la production et l'ingénierie des $V \mathcal{E}$, a proposé des $V \varepsilon$ chargées d'agonistes de nucléotide cyclique (CDN). Les CDN activent la protéine STING (stimulator of interferon genes) [48], dont l'engagement initie la production de cytokines et d'interférons de type I, jouant un rôle majeur dans l'élimination de pathogènes et dans l'établissement d'une immunité anti-tumorale [49] $(\rightarrow)$.

$(\rightarrow)$ Voir la Synthèse de R. Chanut et V. Petrilli, $m / s n^{\circ}$ 6-7,juin-juillet 2019, page 527

L'injection intra-tumorale de CDN libre a été proposée dans plusieurs études précliniques et cliniques, mais leur utilisation reste délicate: l'immunité qui en résulte n'est en effet seulement induite qu'avec une gamme de dose étroite au-delà de laquelle une ablation immunitaire de la tumeur et une inflammation généralisée sont observées. Les $V \varepsilon$, médiateurs de la communication entre cellules tumorales et cellules présentatrices d'antigène, activent la voie de signalisation STING et renforcent l'immunité anti-tumorale. La stratégie envisagée par Codiak Biosciences était de décupler ce potentiel en les chargeant de CDN. Les VE ainsi produites ( $E x O S T I N G$, produites en grande quantité à partir de la lignée HEK293) montrent un efficacité 100 fois supérieure aux CDN libres, en particulier par une meilleure rétention tumorale, une délivrance intracellulaire augmentée et un ciblage spécifique des cellules présentatrices d'antigène dans le microenvironnement tumoral. Cette stratégie pourrait permettre de dépasser les limitations des traitements actuellement à l'essai.

Sébastien Jauliac, à I'hôpital Saint Louis, a montré que des VE issues de cellules cancéreuses peu invasives permettent de limiter le potentiel métastatique de cellules hautement agressives comme les cellules de cancer du sein triple négatif, du pancréas, de mélanome ou de glioblastome [50]. Ces VE issues de cellules cancéreuses mammaires expriment naturellement NFAT3 (nuclear factor of activated T-cells 3 ), un facteur de transcription possédant une activité anti-métastatique : elles inhibent la prolifération et la migration cellulaire, induisent l'apoptose, en collaboration avec des macrophages, et, dans des tumeurs pré-établies, limitent la croissance tumorale et la dissémination de métastases. Des VE aux propriétés anti-métastatiques similaires ont été produites à partir de cellules de la lignée HEK293 modifiées afin qu'elles surexpriment une forme active de NFAT3, ouvrant la voie à une production de masse et à des applications cliniques.

\section{... . et pour d'autres maladies}

Le potentiel des VE à délivrer des protéines est unique, particulièrement dans le cadre de pathologies touchant le système nerveux cen- tral. En fonction de leur origine cellulaire, les $V \varepsilon$ peuvent en effet franchir la barrière hémato-encéphalique [5], 52]. Les maladies de surcharge lysosomale, dues à des défauts d'activité de protéines de lysosomes, sont un exemple d'application pour lesquelles les propriétés des $\checkmark \varepsilon$ pourraient être exploitées. Les protéines lysosomales peuvent être présentes ou enrichies dans des VE qui pourraient les transporter, les protèger, et les délivrer dans différents organes, dont le cerveau, où elles cibleraient naturellement le compartiment lysosomal des cellules réceptrices altérées. De nombreuses maladies lysosomales restent sans traitement. Certaines, comme la maladie de Gaucher ${ }^{7}$ ou la maladie de Fabry ${ }^{8}$, sont traitées par enzymothérapie substitutive, qui consiste en des perfusions intraveineuses à vie d'une enzyme recombinante remplaçant l'enzyme défectueuse ou absente. Malheureusement, une réaction immunitaire contre l'enzyme recombinante peut se produire, conduisant à l'arrêt du traitement.

L'enzyme recombinante n'atteint généralement pas le cerveau et n'a donc pas d'efficacité sur les atteintes cérébrales de la maladie. Récemment, l'équipe d'lbane Abasolo, Val d'Hebron hospital à Barcelone, a montré que des cellules de lignées (CHO DG44 ou HEK293) modifiées afin qu'elles surexpriment l'alpha-galactosidase A (déficiente dans la maladie de Fabry) ou la $\mathrm{N}$-sulfo-glucosamine-sulfohydrolase (déficiente dans la mucopolysaccharidose de type III ou maladie de Sanfillipo) sécrétaient ces enzymes, en grande majorité, dans leurs VE [52]. Après isolement et lyophilisation des VE produites, les enzymes qui restent actives, sont délivrées dans le lysosome des cellules déficientes et restaurent leur fonction lysosomale bien plus efficacement que l'enzyme seule. Chez la souris, ces VE sont retrouvées dans le parenchyme cérébral dès une heure après l'injection intra-artérielle ou intraveineuse, avec une réduction significative de l'accumulation lysosomale de $\mathrm{Gb}^{9}$, ce qui n'est pas observé avec l'enzyme libre. Avec des VE issues de CSM, la délivrance de protéines thérapeutiques dans le cerveau pourrait se combiner à une action anti-inflammatoire, ouvrant la voie à un futur traitement pour les atteintes cérébrales de ces maladies Le Tableau // résume les différents essais cliniques ${ }^{10}$ fondés sur l'utilisation de $V \varepsilon$ chargées [3, 4].

\footnotetext{
${ }^{7}$ La maladie de Gaucher est due à des mutations du gène GBA (1q21) qui code une enzyme lysosomale, la glucosylcéramidase bêta.

${ }^{8}$ La maladie de Fabry est un trouble du métabolisme des glycosphingolipides dû à une activité déficiente (ou absente) de l'alpha-galactosidase $A$ lysosomale liée à des mutations dans le gène GLA (Xq21.3-q22).

9 Le déficit enzymatique responsable de la maladie de Fabry, conduit à l'accumulation des substrats glycosphingolipidiques de l'enzyme, essentiellement le Gb3 (globotriaosylcéramide) et son dérivé déacylé, le lyso-Gb3 ou globotriaosylsphingosine. 10 https://clinicaltrials.gov/ct2/home
} 


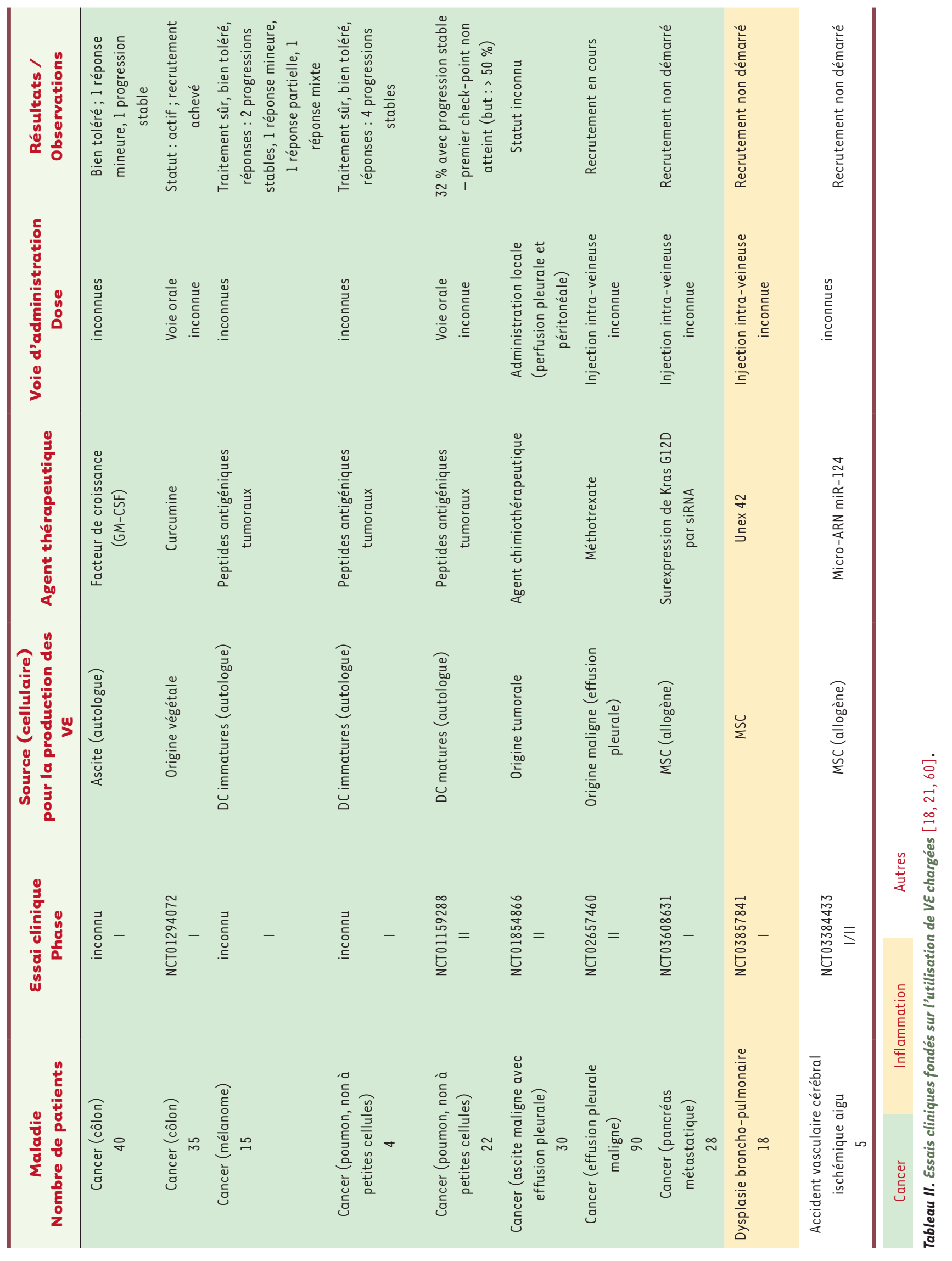




\section{Conclusion}

Les multiples fonctionnalités des vésicules extracellulaires (VE), leurs avantages par rapport aux thérapies cellulaires et aux nanovecteurs synthétiques, les possibilités grandissantes d'ingénierie pour moduler et optimiser leur contenu, leurs activités thérapeutiques originales et leur biodistribution, font des $V \varepsilon$ une biothérapie émergente, versatile et prometteuse pour une très grande variété de maladies. Les défis liés à leur production en masse en condition GMP, au choix et à l'ingénierie des cellules mères (cellules humaines, bactéries, etc.), à leur caractérisation et aux contrôles qualité, aux moyens d'administration, et à la réglementation encadrant leur utilisation, sont en train d'être relevés, à mesure que les connaissances fondamentales, l'innovation et le transfert de technologies progressent.

Si les preuves de concept d'applications sont pléthoriques, seules quelques indications pourront bénéficier rapidement de ces thérapies vésiculaires, si celles-ci démontrent une balance bénéfice/risque avantageuse, un coût abordable et une fenêtre thérapeutique favorable par rapport aux thérapies existantes, ou en cas d'impasse thérapeutique ou d'absence de traitement. Parions ainsi sur leur future application dans le cadre des pathologies orphelines touchant le système nerveux avec une composante inflammatoire. Un des défis du transfert de technologies vers la clinique est de se diriger vers des indications où les thérapies vésiculaires représenteront une avancée majeure et permettront par la suite d'élargir le spectre des indications. $\diamond$

\section{SUMMARY}

\section{Therapeutic applications of extracellular vesicles}

Extracellular vesicles, secreted spontaneously or in response to stress by all cell types, are proposed as alternative biotherapies to cellular therapies and to synthetic nanomedicines. Their logistical advantages (storage, stability, availability, tolerance), their ability to cross biological barriers, to deliver their contents (proteins, lipids and nucleic acids) in order to modify their target cells, as well as their immunomodulatory and regenerative activities, are of growing interest for a very wide spectrum of diseases. Here we review the challenges to bring these biotherapies to the clinic and discuss some promising applications in cancer and regenerative medicine. $\diamond$

\section{LIENS D'INTÉRÊT}

Max Piffoux, Jeanne Gauthier et Amanda K.A. Silva sont co-fondateurs de la start-up Everzom.

Max Piffoux, Amanda K.A. Silva et Florence Gazeau sont co-fondateurs de la start-up Evora Biosciences.

Kelly Aubertin et Anna Sebbagh déclarent n'avoir aucun lien d'intérêt concernant les données publiées dans cet article

\section{RÉFÉRENCES}

1. Verma M, Lam TK, Hebert $\varepsilon$, et al. Extracellular vesicles: potential applications in cancer diagnosis, prognosis, and epidemiology. BMC Clin Pathol $2015 ; 15$.

2. Saint-Pol J, Gosselet F, Duban-Deweer S, et al. Targeting and Crossing the Blood-Brain Barrier with Extracellular Vesicles. Cells $2020 ; 9: 851$.

3. Wiklander OPB, Brennan MÁ, Lötvall J, et al. Advances in therapeutic applications of extracellular vesicles. Sci Transl Med $2019 ; 11$ : eaav8521.
4. Nagelkerke A, Ojansivu M, Koog $L$ van der, et al. Extracellular vesicles for tissue repair and regeneration: evidence, challenges and opportunities. Adv Drug Deliv Rev 2021 ; 175 : 113775.

5. Elsharkasy OM, Nordin JZ, Hagey DW, et al. Extracellular vesicles as drug delivery systems: Why and how? Adv Drug Deliv Rev 2020 ; 159 : 332-43.

6. Lener T, Gimona M, Aigner L, et al. Applying extracellular vesicles based therapeutics in clinical trials - an ISEV position paper. J Extracell Vesicles $2015 ; 4$.

7. Théry C, Witwer KW, Aikawa $\varepsilon$, et al. Minimal information for studies of extracellular vesicles 2018 (MISEV2018): a position statement of the International Society for Extracellular Vesicles and update of the MISEV2014 guidelines. J Extracell Vesicles 2018 ; $7: 1535750$.

8. Rohban R, Pieber TR. Mesenchymal Stem and Progenitor Cells in Regeneration: Tissue Specificity and Regenerative Potential. Stem Cells Int 2017 ; 2017 : e5173732.

9. Grangier A, Branchu J, Volatron J, et al. Technological advances towards extracellular vesicles mass production. Adv Drug Deliv Rev 2021 ; 113843

10. Piffoux M, Nicolás-Boluda A, Mulens-Arias V, et al. Extracellular vesicles for personalized medicine: The input of physically triggered production, loading and theranostic properties. Adv Drug Deliv Rev 2019 ; 138 : 247-58.

11. Vion Anne-Clémence, Ramkhelawon Bhama, Loyer Xavier, et al. Shear Stress Regulates Endothelial Microparticle Release. Circul Res 2013 ; 112 : 1323-33.

12. Pinto A, Marangon I, Méreaux J, et al. Immune Reprogramming Precision Photodynamic Therapy of Peritoneal Metastasis by Scalable Stem-CellDerived Extracellular Vesicles. ACS Nano 2021 ; 15 : 3251-63.

13. Berger A, Araújo-Filho I, Piffoux M, et al. Local administration of stem cellderived extracellular vesicles in a thermoresponsive hydrogel promotes a pro-healing effect in a rat model of colo-cutaneous post-surgical fistula. Nanoscale $2021 ; 13: 218-32$.

14. Aubertin K, Silva AKA, Luciani N, et al. Massive release of extracellular vesicles from cancer cells after photodynamic treatment or chemotherapy. Sci Rep $2016 ; 6$ : 35376.

15. Keklikoglou I, Cianciaruso C, Güç $\varepsilon$, et al. Chemotherapy elicits prometastatic extracellular vesicles in breast cancer models. Nat Cell Biol $2019 ; 21: 190-202$.

16. Gauthier NC, Fardin MA, Roca-Cusachs P, et al. Temporary increase in plasma membrane tension coordinates the activation of exocytosis and contraction during cell spreading. Proc Natl Acad Sci U S A 2011 ; 108 : 14467-14472.

17. Bodin P, Burnstock G. Evidence That Release of Adenosine Triphosphate From Endothelial Cells During Increased Shear Stress Is Vesicular. J Cardiovasc Pharmacol $2001 ; 38: 900-8$.

18. Jong $O G$ de, Verhaar MC, Chen $Y$, et al. Cellular stress conditions are reflected in the protein and RNA content of endothelial cell-derived exosomes. J Extracell Vesicles $2012 ; 1: 18396$.

19. Park K-S, Bandeira $\varepsilon$, Shelke GV, et al. Enhancement of therapeutic potential of mesenchymal stem cell-derived extracellular vesicles. Stem Cell Res Ther $2019 ; 10: 288$.

20. Staubach S, Bauer FN, Tertel T, et al. Scaled preparation of extracellular vesicles from conditioned media. Adv Drug Deliv Rev 2021; $177: 113940$.

21. Gupta D, Maria Zickler A, EL Andaloussi S. Dosing Extracellular Vesicles. Adv Drug Deliv Rev $2021 ; 113961$.

22. Bonsergent $\varepsilon$, Grisard $\varepsilon$, Buchrieser J, et al. Quantitative characterization of extracellular vesicle uptake and content delivery within mammalian cells. Nat Commun 2021; 12 : 1864.

23. Balkom BWM van, Gremmels H, Giebel B, et al. Proteomic Signature of Mesenchymal Stromal Cell-Derived Small Extracellular Vesicles. Proteomics $2019 ; 19: 1800163$.

24. Sebbagh AC, Rosenbaum B, Péré G, et al. Regenerative medicine for digestive fistulae therapy: benefits, challenges and promises of stem/ stromal cells and emergent perspectives via their extracellular vesicles. Adv Drug Deliv Rev 2021 ; 113841.

25. Maumus M, Pers Y-M, Ruiz M, et al. Cellules souches mésenchymateuses et médecine régénératrice - Quel avenir pour l'arthrose ? Med Sci (Paris) 2018 ; 34 : 1092-9.

26. Kordelas L, Rebmann V, Ludwig A-K, et al. MSC-derived exosomes: a novel tool to treat therapy-refractory graft-versus-host disease. Leukemia 2014 ; $28: 970-73$.

27. Doeppner TR, Herz J, Görgens A, et al. Extracellular Vesicles Improve PostStroke Neuroregeneration and Prevent Postischemic Immunosuppression. Stem Cells Transl Med $2015 ; 4: 1131-43$. 


\section{RÉFÉRENCES}

28. Liao Z, Liu H, Ma L, et al. Engineering Extracellular Vesicles Restore the Impaired Cellular Uptake and Attenuate Intervertebral Disc Degeneration. ACS Nano 2021 ; 15(9) : 14709-24.

29. Lai RC, Arslan F, Lee MM, et al. Exosome secreted by MSC reduces myocardial ischemia/reperfusion injury. Stem Cell Res $2010 ; 4: 214-22$.

30. Clement O, Cellier C, Wilhelm C, et al. Compositions useful for mucosal healing. W0/2018/011192.

31. Silva AKA, Perretta S, Perrod G, et al. Thermoresponsive Gel Embedded with Adipose Stem-CellDerived Extracellular Vesicles Promotes Esophageal Fistula Healing in a Thermo-Actuated Delivery Strategy. ACS Nano $2018 ; 12: 9800-14$.

32. Berger A, Caudron $\varepsilon$, Perrod $G$, et al. Enhancing digestive fistula healing by the off-label use of a thermoresponsive vessel occluder polymer associated with esophageal stent placement: A case report. Clin Res Hepatol Gastroenterol 2021 ; 45 : 101474.

33. Coffin $\varepsilon$, Grangier A, Perrod G, et al. Extracellular vesicles from adipose stromal cells combined with a thermoresponsive hydrogel prevent esophageal stricture after extensive endoscopic submucosal dissection in a porcine model. Nanoscale $2021 ; 13: 14866-78$.

34. Kervadec A, Bellamy V, દl Harane N, et al. Cardiovascular progenitor-derived extracellular vesicles recapitulate the beneficial effects of their parent cells in the treatment of chronic heart failure.J Heart Lung Transplant 2016 ; $35: 795-807$.

35. Gao L, Wang L, Wei Y, et al. Exosomes secreted by hiPSC-derived cardiac cells improve recovery from myocardial infarction in swine. Sci Transl Med $2020 ; 12$ : eaayl318.

36. El Harane N, Kervadec A, Bellamy V, et al. Acellular therapeutic approach for heart failure: in vitro production of extracellular vesicles from human cardiovascular progenitors. Eur Heart J 2018 ; $39: 1835-47$.

37. Lima Correa B, દl Harane N, Gomez I, et al. Extracellular vesicles from human cardiovascular progenitors trigger a reparative immune response in infarcted hearts. Cardiovascul Res 2021 ; $117: 292-307$.

38. Cosenza S, Ruiz M, Toupet $K$, et al. Mesenchymal stem cells derived exosomes and microparticles protect cartilage and bone from degradation in osteoarthritis. Sci Rep $2017 ; 7: 16214$.

39. Cosenza S, Toupet K, Maumus M, et al. Mesenchymal stem cells-derived exosomes are more immunosuppressive than microparticles in inflammatory arthritis. Theranostics $2018 ; 8: 1399$ 410.

40. Rozier P, Maumus M, Maria ATJ, et al. Mesenchymal stromal cells-derived extracellular vesicles alleviate systemic sclerosis via miR-29a-3p. J Autoimmun 2021; 121 : 102660.

41. Warnecke A, Prenzler N, Harre J, et al. First-in-human intracochlear application of human stromal cell-derived extracellular vesicles. J Extracell Vesicles $2021 ; 10$ : el2094.

42. Warnecke A, Harre J, Staecker $\mathrm{H}$, et al. Extracellular vesicles from human multipotent stromal cells protect against hearing loss after noise trauma in vivo. Clin Transl Med $2020 ; 10:$ e262
43. Piffoux M, Volatron J, Cherukula K, et al. Engineering and loading therapeutic extracellular vesicles for clinical translation: a data reporting frame for comparability. Adv Drug Deliv Rev 2021 ; 113972.

44. Piffoux M, Silva AKA, Wilhelm C, et al. Modification of Extracellular Vesicles by Fusion with Liposomes for the Design of Personalized Biogenic Drug Delivery Systems. ACS Nano $2018 ; 12: 6830-42$.

45. Toledano Furman NE, Lupu-Haber $Y$, Bronshtein T, et al. Reconstructed Stem Cell Nanoghosts: A Natural Tumor Targeting Platform. Nano Lett 2013 ; 13 : 3248-55.

46. Silva AKA, Kolosnjaj-Tabi J, Bonneau S, et al. Magnetic and Photoresponsive Theranosomes: Translating Cell-Released Vesicles into Smart Nanovectors for Cancer Therapy. ACS Nano $2013 ; 7$ : 4954-66.

47. Yakavets I, Francois A, Lamy L, et al. Effect of stroma on the behavior of temoporfin-loaded lipid nanovesicles inside the stroma-rich head and neck carcinoma spheroids. J Nanobiotech $2021 ; 19: 3$.

48. Jang SC, Economides KD, Moniz RJ, et al. ExoSTING, an extracellular vesicle loaded with STING agonists, promotes tumor immune surveillance. Commun Biol $2021 ; 4$ : 1-17.

49. Chanut R, Petrilli V. Détection de I'ADN cytosolique par la voie cGAS-STING - De l'immunité innée vers le contrôle de la tumorigenèse. Med Sci (Paris) $2019 ; 35: 527-34$

50. Camargo LCB de, Guaddachi F, Bergerat D, et al. Extracellular vesicles produced by NFAT3-expressing cells hinder tumor growth and metastatic dissemination. Sci Rep $2020 ; 10: 8964$

51. Perets $\mathrm{N}$, Oron 0 , Herman $\mathrm{S}$, et al. Exosomes derived from mesenchymal stem cells improved core symptoms of genetically modified mouse model of autism Shank3B. Molecular Autism 2020; 11 : 65.

52. Seras-Franzoso J, Díaz-Riascos ZV, Corchero JL, et al. Extracellular vesicles from recombinant cell factories improve the activity and efficacy of enzymes defective in lysosomal storage disorders. J Extracell Vesicles 2021 10 : el2058.

53. Silva AKA, Morille M, Piffoux M, et al. Development of extracellular vesiclebased medicinal products: a position paper of the group Extracellular Vesicle translation to clinicaL perspectiVEs - EVOLVE France. Adv Drug Deliv Rev $2021 ; 179$ : 114001 .

\section{TIRÉS À PART}

F. Gazeau

P ossédées du Malin au Moyen-Âge, les sorcières hystériques sont vouées au bûcher. Enfermées au XvII ${ }^{e}$ siècle, maltraitées, elles rejoignent la Cour des Miracles de l'Hospice de la Vieillesse-Femmes à la Salpêtrière... Jusqu'à ce que le Dr JeanMartin Charcot (1825-1893) mène le combat qui transforme l'ancien hospice en hôpital : l'École de la Salpêtrière de

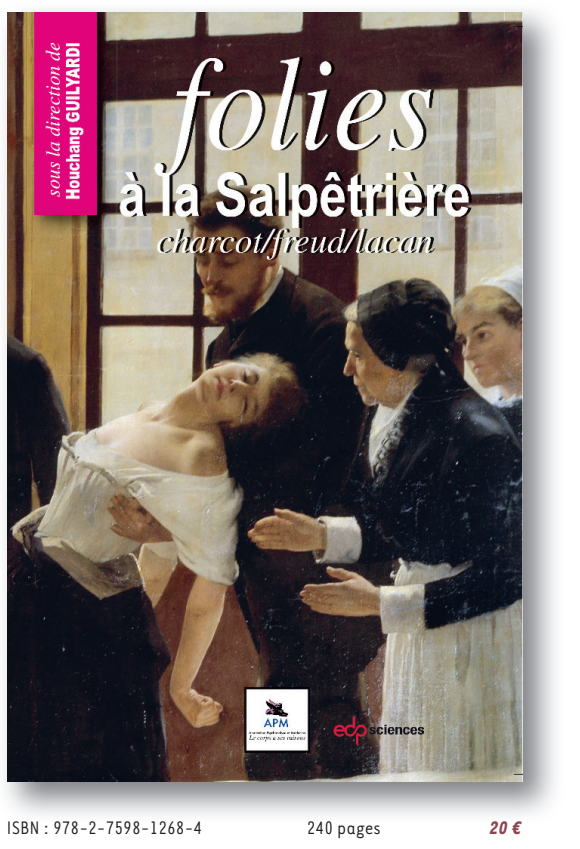
Paris est née, qui devient lieu de recherche, d'enseignement et de soins, de renommée internationale.

Jean Martin Charcot n'a pas bonne presse, et pourtant... Hystérie et folie traversent les siècles, prenant les formes de «l'air du temps ».

De l'utérus migrateur d'Hippocrate aux recherches neurologiques de Charcot. Du désir inconscient avec Freud à la jouissance du parlêtre chez Lacan... C'est à cette traversée historique et conceptuelle que nous convie cet ouvrage.

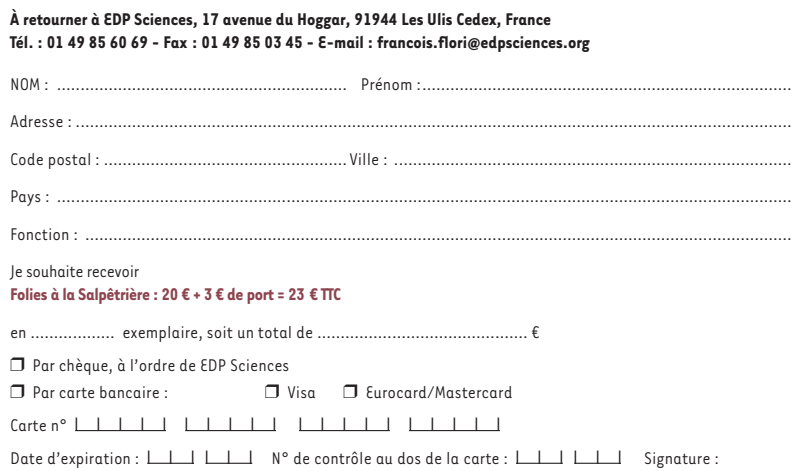

